\title{
Local structure analysis by pair distribution function obtained from a TEM
}

\author{
C. W. Tai \\ Department of Materials and Environmental Chemistry, Stockholm University, Stockholm, SE-10691 Sweden \\ cheuk-wai.tai@mmk.su.se
}

The pair-distribution function (PDF) method is widely used to obtain structural information beyond the typical diffraction techniques together with standard structure refinement utilizing Bragg reflections [1]. PDF analysis using x-ray and neutron powder diffraction data is well established. Electron-based PDF (ePDF) analysis has drawn considerable attention in recent years $[2,3,4]$. In addition to the very strong electron-matter interaction $\left(\sim 10^{5}\right.$ than $\mathrm{x}$-ray $)$, the main advantages of the electron-based over $\mathrm{x}$-ray and neutron-based is to utilize modern electron microscopes, which offer (sub)nano-sized probe of the electron beam and various imaging and spectroscopy techniques simultaneously. Therefore, ePDF is particularly good for study nano-materials, disordered materials and specific region of interest in the specimens.

Although the procedures of ePDF analysis is similar to those obtained by x-ray and neutron, several parameters and steps, which are due to electron scattering and TEM practice, are crucial in the processing. For instance, $\mathrm{Q}_{\max }$ is always important in all PDF experiments. However, $Q_{\min }$, which is not considered in x-ray and neutron, should be carefully determined in ePDF. On the other hand, the shape factor can influence the ePDF results. The smaller the particles the stronger effect can be seen. Different stepwise atomic layer arrangement of the crystal surface can contribute significantly in the analysis [5]. In addition to experimental and analysis procedures different to those for x-ray or neutron-based, the uniqueness and possibility of ePDF analyses of some amorphous materials and nanostructures will be discussed.

[1] Egami, T. \& Billinge, S. J. L. (2002). Underneath the Bragg Peaks: Structural Analysis of Complex Materials. Amsterdam: Elsevier Science.

[2] Abeykoon, M., Malliakas, C. D., Juhás, D., Božin, E. S., Kanatzidis, M. G. \& Billinge, S. J. L. (2012). Z. Kristallogr. 227248.

[3] Tran, D.-T., Svensson, G. \& Tai, C.-W. (2017). J. Appl. Crystallogr. 50, 304.

[4] Gorelik, T. E., Neder, R., Terban, M. W., Lee, Z., Mu, X., Jung, C., Jacob T. \& Kaiser, U. (2019) Acta Crystallogr. B 75, 532.

[5] Tran, D.-T., Svensson, G. \& Tai, C.-W. (2016). arXiv:1602.08078.

\section{Keywords: pair distribution function, electron diffraction, electron crystallography, TEM, disorder}

The Knut and Alice Wallenberg (KAW) Foundation is acknowledged for providing the electron microscopy facilities and financial support under the project 3DEM-NATUR for the initial development. Swedish Research Council (project no. 2018-05260) is also acknowledged. 